\title{
C1q tumor necrosis factor-related protein-3 protects mesenchymal stem cells against hypoxia- and serum deprivation-induced apoptosis through the phosphoinositide 3-kinase/Akt pathway
}

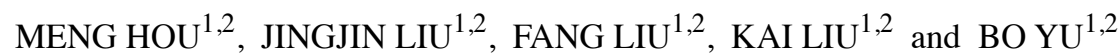 \\ ${ }^{1}$ The Key Laboratory of Myocardial Ischemia, Harbin Medical University, Ministry of Education, Heilongjiang Province; \\ ${ }^{2}$ Department of Cardiology, The Second Affiliated Hospital of Harbin Medical University, Harbin, Heilongjiang, P.R. China
}

Received August 1, 2013; Accepted October 24, 2013

DOI: $10.3892 /$ ijmm.2013.1550

\begin{abstract}
Bone marrow (BM)-derived mesenchymal stem cells (MSCs) represent the leading candidate cell for tissue regeneration in the ischemic myocardium. However, the poor survival of stem cells transplanted into the ischemic myocardium presents a major obstacle in stem cell-based therapy. Clq tumor necrosis factor-related protein 3 (CTRP3) is a newly identified adipokine, similar to adiponectin, with beneficial effects on metabolic regulation. It has been shown to enhance the survival of cardiomyocytes during ischemia, while its expression is reduced following ischemia. In the present study, we examined the hypothesis that CTRP 3 may enhance the survival of MSCs during exposure to hypoxia/serum deprivation (SD), and attempted to elucidate the underlying mechanisms. MSCs were obtained from rat bone marrow and cultured. Apoptosis was induced by hypoxia/SD for up to $24 \mathrm{~h}$ and the apoptotic rates were assessed by flow cytometry. MSC proliferation was measured using a Cell Counting kit-8 assay. The expression levels of Akt, Bcl-2, Bax, cytochrome $c$ and cleaved caspase-3 were detected by western blot analysis. Mitochondrial membrane potential was examined using a membrane-permeable dye. CTRP3 significantly reduced hypoxia/SD-induced apoptosis in a concentration-dependent manner. The hypoxia/SD-induced decrease in the Bcl-2/Bax ratio and the mitochondrial membrane potential, and the increase in cytochrome $c$ and caspase-3 levels were largely reversed by CTRP3. The anti-apoptotic effects of CTRP3 were blocked by inhibiting the activation of phosphoinositide 3-kinase (PI3K)/Akt signaling pathway with the PI3K inhibitor,LY294002. In conclusion, CTRP3 is a novel anti-apoptotic adipokine that protects MSCs from hypoxia/SD-induced apoptosis through the PI3K/Akt signaling pathway.
\end{abstract}

Correspondence to: Dr Bo Yu, Department of Cardiology, The Second Affiliated Hospital of Harbin Medical University, 148 Baojian Road, Harbin, Heilongjiang 150086, P.R. China

E-mail: yubodr@163.com

Key words: Clq tumor necrosis factor-related protein-3, hypoxia, mesenchymal stem cell, apoptosis, phosphoinositide 3-kinase/Akt pathway, serum deprivation

\section{Introduction}

Ischemia/reperfusion injury remains a leading cause of mortality in developed countries, despite significant advances in the medical treatment of heart failure (1). A number of investigators have demonstrated that the transplantation of bone marrow (BM)-derived mesenchymal stem cells (MSCs) represents a promising tool for the repair and regeneration of cardiomyocytes and for the restoration of heart function (2-4). However, the poor survival of engrafted MSCs presents a major obstacle in MSC-based therapy, and evidence suggests that the transplanted cells undergo apoptosis $(5,6)$. Previous studies have demonstrated that hypoxia and serum deprivation (SD), which are both components of ischemia, induce MSC apoptosis through the mitochondrial pathway $(7,8)$. Strategies that enhance tolerance to apoptosis should thus significantly improve the efficacy of MSC-based transplantation therapy.

Clq tumor necrosis factor-related proteins (CTRPs) are members of the highly conserved family of adiponectins. Each of the 10 known members (CTRP1-CTRP10) consists of 4 distinct domains, including an $\mathrm{N}$-terminal signal peptide, a short variable domain, a collagen-like domain and a C-terminal Clq-like globular domain $(9,10)$. CTRP3 is expressed not only in cartilage, where it was originally discovered, but also in adipose tissue, preadipocyte cell lines, as well as in monocytes and fibroblasts, and has been shown to be upregulated upon fasting and inflammation (11-13). CTRP3, originally thought to participate in lipid metabolism, has broad functions and regulates various biological processes, including adipokine secretion, fatty acid oxidation, inflammation, cell proliferation, differentiation and apoptosis (14-17). CTRP3 protects cardiomyocytes from apoptosis and plays a protective role in cardiac infarction (17). Furthermore, recent findings have suggested that CTRP3 acts via the phosphoinositide 3-kinase (PI3K)/Akt pathway to exert a marked anti-apoptotic effect (17). The PI3K/Akt pathway is involved in protecting numerous cell types, including MSCs, from apoptosis. It is therefore possible that CTRP3 may be involved in protecting MSCs against hypoxia/SD-induced apoptosis. CTRP3 and the related downstream signaling pathway(s) may thus represent promising targets for preventing hypoxia/SD-induced apoptosis. Therefore, the present study aimed to examine the 
effects of CTRP3 on hypoxia/SD-induced MSC apoptosis and to investigate the related signaling pathways.

\section{Materials and methods}

Animals. Male Sprague-Dawley rats, weighing 60-80 g, were handled in accordance with the US National Institutes of Health published guidelines. All procedures were approved by the Institutional Animal Care and Use Committee of Harbin Medical University. The study was conducted in compliance with the Guide for the Care and Use of Laboratory Animals published by the National Institutes of Health (NIH, revised in 1996).

Reagents. Dulbecco's modified Eagle's medium (DMEM)/F12 and fetal bovine serum (FBS) were purchased from HyClone Laboratories (Logan, UT, USA). The Annexin V-FITC apoptosis detection kit, and anti-CD44, anti-CD29 and anti-CD90 antibodies were obtained from BD Biosciences (San Diego, CA, USA). Anti-CD34 and anti-CD45 antibodies were obtained from eBioscience (San Diego, CA, USA). Rabbit monoclonal antibodies against Akt, phospho-Akt (Tyr308, Ser473), caspase-3, Bax, $\mathrm{Bcl}-2$ and cytochrome $c$, and the PI3K inhibitor, LY294002, were obtained from Cell Signaling Technology (Danvers, MA, USA). Mouse polyclonal antibody against $\beta$-actin (\#TA-09) was purchased from Zhongshan Goldenbridge Biotechnology (Beijing, China) and horseradish peroxidaseconjugated secondary antibodies anti-mouse and -rabbit were obtained from Santa Cruz Biotechnology, Inc. (Santa Cruz, CA, USA). The JC-1 mitochondrial membrane potential assay kit was purchased from the Beyotime Institute of Biotechnology (Nantong, China), the Cell Counting kit-8 (CCK-8) assay kit was from HaiGene Technology (Harbin, China) and recombinant human CTRP3 was from Adipogen (San Diego, CA, USA).

Cell cultures and treatments. BM-MSCs were isolated from the femurs and tibias of Sprague-Dawley rats, as previously described (18). Briefly, BM cells were flushed from the femurs and tibias with $5 \mathrm{ml}$ DMEM/F12. Red blood cells were lysed and removed, and $5 \times 10^{5}$ cells were then plated in a $25-\mathrm{cm}^{2}$ flask with $6 \mathrm{ml}$ DMEM/F12 supplemented with 10\% FBS and $1 \%$ penicillin/streptomycin. After 3 days of culture at $37^{\circ} \mathrm{C}$ under $5 \% \mathrm{CO}_{2}$, the medium and non-adherent cells were removed and replaced with fresh medium. Adherent MSCs were further grown in medium, which was replaced every 3 days. When the cells reached $80-90 \%$ confluence, the adherent cells were trypsinized and expanded at 2:3 or 1:2 dilutions. All cells used were from passages 3-5.

The characteristics of the MSCs were determined by immunophenotyping. Cells were harvested, washed with phosphate-buffered saline (PBS), and labeled with phycoerythrin (PE)-conjugated anti-CD45 and anti-CD90 antibodies, and fluorescein isothiocyanate (FITC)-labeled anti-CD44, anti-CD29 and anti-CD34 antibodies. Labeled cells were assayed by flow cytometry and analyzed using FACSDiva Pro software (BD Biosciences).

The in vitro induction of apoptosis by hypoxia/SD was designed to mimic the in vivo conditions in the ischemic myocardium, and was initiated as previously described (19). Cells exposed to hypoxia/SD alone were used as the apoptotic controls. Apoptosis was induced by incubating the MSCs in serum-free medium in a controlled (anaerobic)-atmosphere glove chamber (855-AC; Plas-Labs Inc., Lansing, MI, USA), so as to scavenge free oxygen. Cells cultured in complete medium alone were used as the non-ischemic controls. Cells were exposed to CTRP3 when subjected to hypoxia/SD. CTRP3 concentrations of $3-3,000 \mathrm{ng} / \mathrm{ml}$ were used to treat the cells throughout the process.

To investigate the involvement of the PI3K/Akt pathway, the cells were pre-incubated with the PI3K/Akt inhibitor, LY294002 $(25 \mu \mathrm{M})$, in complete medium for $90 \mathrm{~min}$ prior to exposure to hypoxia/SD. CTRP3 was added in the presence of the inhibitor during exposure to hypoxia/SD.

Analysis of cell apoptosis by flow cytometry. Apoptotic rates were estimated by detecting phosphatidylserine on the cell plasma membrane using the fluorescent dye [propidium iodide (PI)] with the Annexin V-FITC apoptosis detection kit (BD Biosciences), according to the manufacturer's instructions. In brief, cells were harvested and washed in ice-cold PBS, resuspended in $300 \mu \mathrm{l}$ of binding buffer and incubated with $5 \mu \mathrm{l}$ of Annexin V-FITC solution for $30 \mathrm{~min}$ at $4^{\circ} \mathrm{C}$ in the dark, followed by further incubation with $5 \mu 1$ PI for 5 min. Cells were then analyzed immediately by bivariate flow cytometry using a BD FACSCanto cytometer equipped with FACSDiva Pro software. Approximately $1-5 \times 10^{5}$ cells were analyzed in each sample.

Cell proliferation assay. Cell proliferation was assessed using a CCK-8 assay kit according to the manufacturer's instructions. Cells were incubated with CCK-8 solution in 96-well plates for $1 \mathrm{~h}$ at $37^{\circ} \mathrm{C}$. The absorbance of each well was quantified at $450 \mathrm{~nm}$.

Toxicity assay. The potential toxic effects of CTRP 3 in cultured MSCs were examined at various concentrations. The MSCs were incubated in culture medium supplemented with CTRP3 for $24 \mathrm{~h}$ under hypoxia/SD culture conditions, and for 3 days under normal culture conditions. Trypan blue was added to the medium and the cells were incubated at $37^{\circ} \mathrm{C}$ for $15 \mathrm{~min}$. Trypan blue-positive cells were then counted under a phase-contrast microscope. Five fields were randomly selected for each dish and at least 3 dishes were counted for each concentration.

Western blot analysis. Western blot analysis was carried out as previously described (20). Briefly, cells were washed twice with ice-cold PBS and ruptured with lysis buffer containing $20 \mathrm{mM}$ Tris- $\mathrm{HCl}, 150 \mathrm{mM} \mathrm{NaCl}, 1 \%$ Triton X-100, protease and phosphatase inhibitors. Cell extracts were centrifuged for $5 \mathrm{~min}$ at $12,000 \mathrm{x} \mathrm{g}$ and supernatants were collected. A total of $20 \mu \mathrm{g}$ of protein was resolved by SDS-PAGE and transferred onto PVDF membranes. Membranes were blocked for $1 \mathrm{~h}$ with 5\% skim milk in Tris-buffered saline solution containing $0.1 \%$ Tween-20 and were incubated with primary antibodies [rabbit monoclonal antibodies against Akt, phospho-Akt (Tyr308, Ser473), caspase-3, Bax, Bcl-2, cytochrome $c$ and mouse polyclonal antibody against $\beta$-actin] at $4^{\circ} \mathrm{C}$ overnight. The membranes were washed, incubated for $1 \mathrm{~h}$ with the appropriate horseradish peroxidase-conjugated secondary antibodies (anti-mouse and anti-rabbit), developed using chemiluminescent substrates, photographed using ChemiDoc 
A

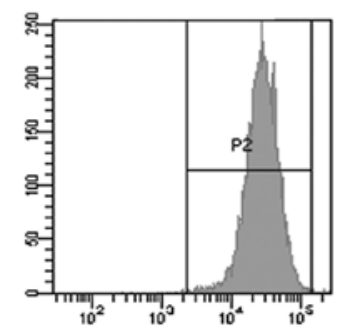

CD29

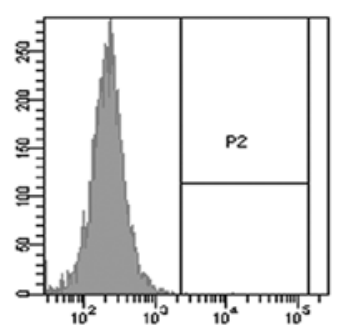

CD34

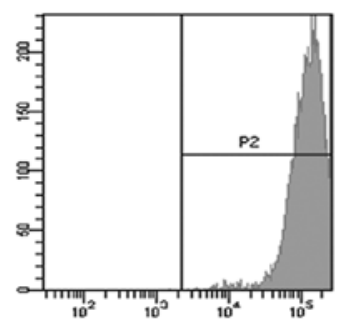

CD90

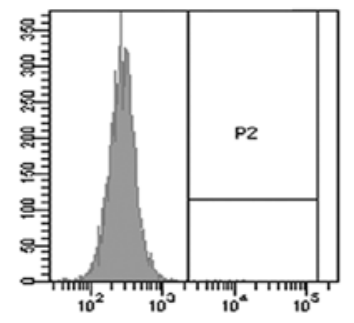

CD45

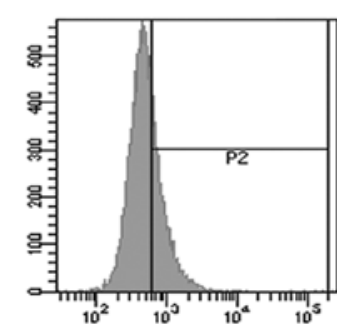

CD44

B

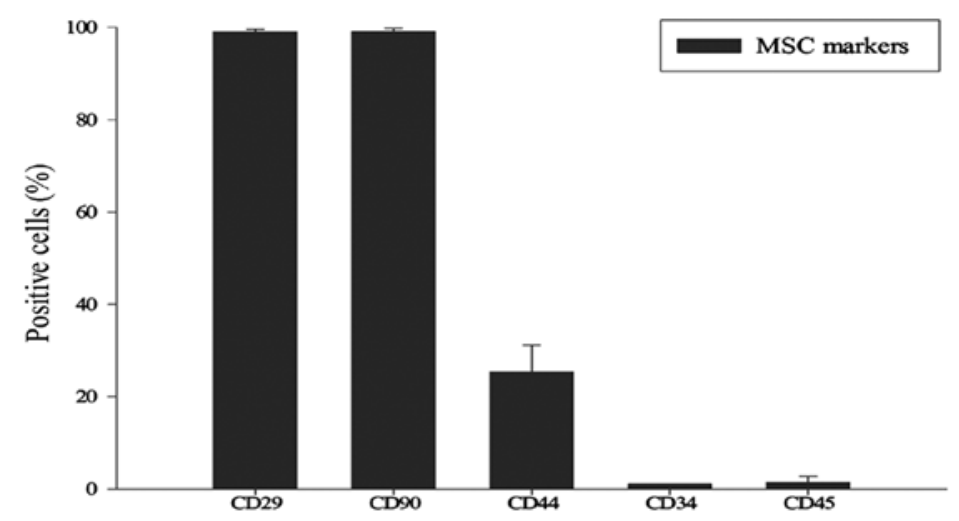

Figure 1. Cell surface markers of mesenchymal stem cells (MSCs). Surface marker molecules expressed in passage 3 MSCs were (A) analyzed by flow cytometry and (B) quantified by the image analysis of positive cells.

XRS equipment (Bio-Rad, Hercules, CA, USA) and quantified using Quantity One software (Bio-Rad).

Mitochondrial membrane potential assay. Mitochondrial membrane potential was determined using the JC-1 mitochondrial membrane potential assay kit. Briefly, the MSCs were seeded in 6-well plates. Following treatment, the cells were washed twice with PBS, and $1 \mathrm{ml}$ staining dye/well was added (culture medium:JC-1 working dye, 1:1) and incubated at $37^{\circ} \mathrm{C}$ for $20 \mathrm{~min}$. The cells were then washed twice with cold JC-1 staining buffer and examined under a fluorescence microscope.

Statistical analysis. Data are expressed as the means \pm standard deviation (SD). Differences among groups were tested by a one-way ANOVA. Comparisons between groups were evaluated using the Student's t-test. A value of $\mathrm{P}<0.05$ was considered to indicate a statistically significant difference.

\section{Results}

Cells isolated from rat BM show MSC characteristics. MSCs were uniformly spindle-like. FACS analysis revealed that the majority of cells from passage 3 expressed the common MSC surface markers, CD29 (98.98 $\pm 0.55 \%)$, CD90 (99.06 $\pm 0.63 \%)$ and CD44 (25.30 $\pm 5.80 \%)$, but were found negative for CD34 $(0.97 \pm 0.03 \%)$ and CD45 (1.38 $\pm 1.30 \%)$ (Fig. 1). Cells cultured in vitro for 3-5 passages were therefore used in the subsequent experiments.

CTRP3 protects MSCs from hypoxia/SD-induced apoptosis. In preliminary experiments, the maximal induction of early apoptosis by hypoxia/SD in the MSCs occurred at $24 \mathrm{~h}$. We investigated whether CTRP3 protects MSCs from this process. MSCs were exposed to increasing concentrations of CTRP3 (3-3,000 ng/ml) followed by exposure to hypoxia/SD for $24 \mathrm{~h}$, and cell apoptosis was determined by FACS analysis. CTRP3 (3-3,000 ng/ml) effectively blocked the apoptotic process, and the ratio of apoptotic cells decreased with a pronounced effect at $300 \mathrm{ng} / \mathrm{ml}$ [treated cells, $2.60 \pm 0.23$ vs. apoptotic control (untreated cells), 6.95 \pm 0.37 ] (Fig. 2A and B).

Caspase-3 is a key mediator of apoptosis. We further evaluated the anti-apoptotic effects of CTRP 3 by western blot analysis using a caspase- 3 monoclonal antibody, and confirmed that CRTP3 significantly inhibited the cleavage of caspase-3 in a concentration-dependent manner following exposure to 

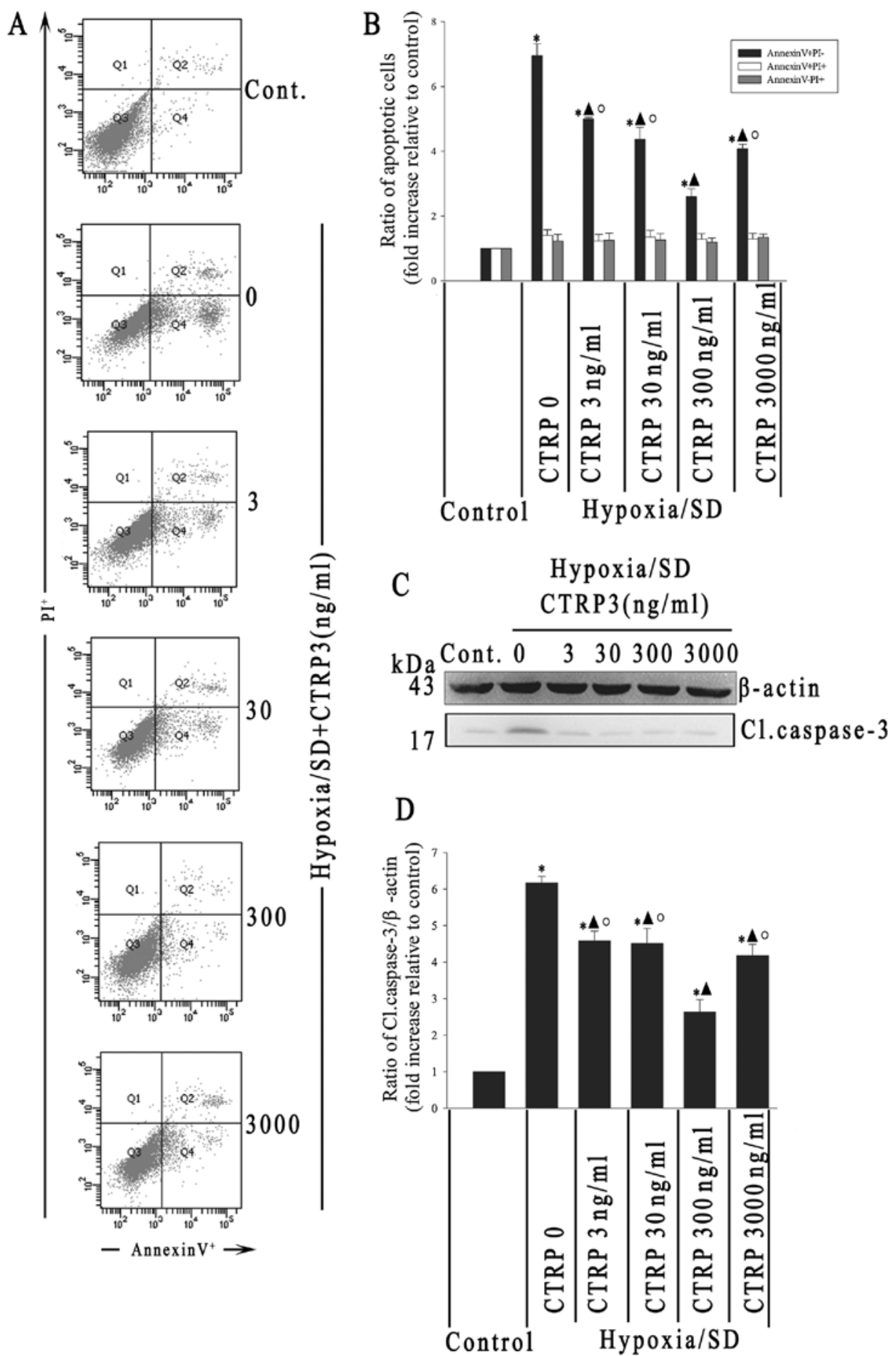

Figure 2. Clq tumor necrosis factor-related protein 3 (CTRP3) protects mesenchymal stem cells (MSCs) against hypoxia/serum deprivation (SD)-induced apoptosis. CTRP3 (3-3,000 ng/ml) was added at the time point of exposure to hypoxia/SD and was maintained in the incubation medium throughout the hypoxia/SD treatment period. Apoptosis was measured by flow cytometry. (A) Representative plots from 3 FACScan flow cytometric analyses of apoptotic cells following Annexin V and PI staining. (B) Fold changes of cell apoptosis relative to control cells. Each column represents the mean \pm SD of 3 independent experiments. (C) Western blot analysis using the caspase-3 antibody to monitor the cleavage of caspase-3 induced by hypoxia/SD, and the inhibition of the cleavage of caspase- 3 by various concentrations of CTRP3. The blot is representative of 3 independent experiments. (D) Fold changes of cleavage of caspase-3 relative to $\beta$-actin. Each column represents the mean $\pm \mathrm{SD}$ of 3 independent experiments. (A and C) Cont., control, (B and D) ${ }^{*} \mathrm{P}<0.05$ vs. control; ${ }^{\wedge} \mathrm{P}<0.05$ vs. hypoxia/SD; ${ }^{\circ} \mathrm{P}<0.05$ vs. hypoxia/SD + CTRP3 (300 ng/ml). Cl. caspase-3, cleaved caspase-3. Control, non-ischemic controls; CTRP 0, apoptotic controls (untreated cells).

hypoxia/SD (apoptotic control, $6.17 \pm 0.17$ vs. treated cells, $2.63 \pm 0.34$ ) (Fig. 2C and D).

To the best of our knowledge, the potential toxicity of CTRP3 to MSCs at the tested concentrations has not been previously addressed. We therefore examined the effects of CTRP3 on MSC viability. Trypan blue assays indicated that up to $3,000 \mathrm{ng} / \mathrm{ml}$ of CTRP3 had no adverse effects on the viability of MSCs (Fig. 3A and B). 

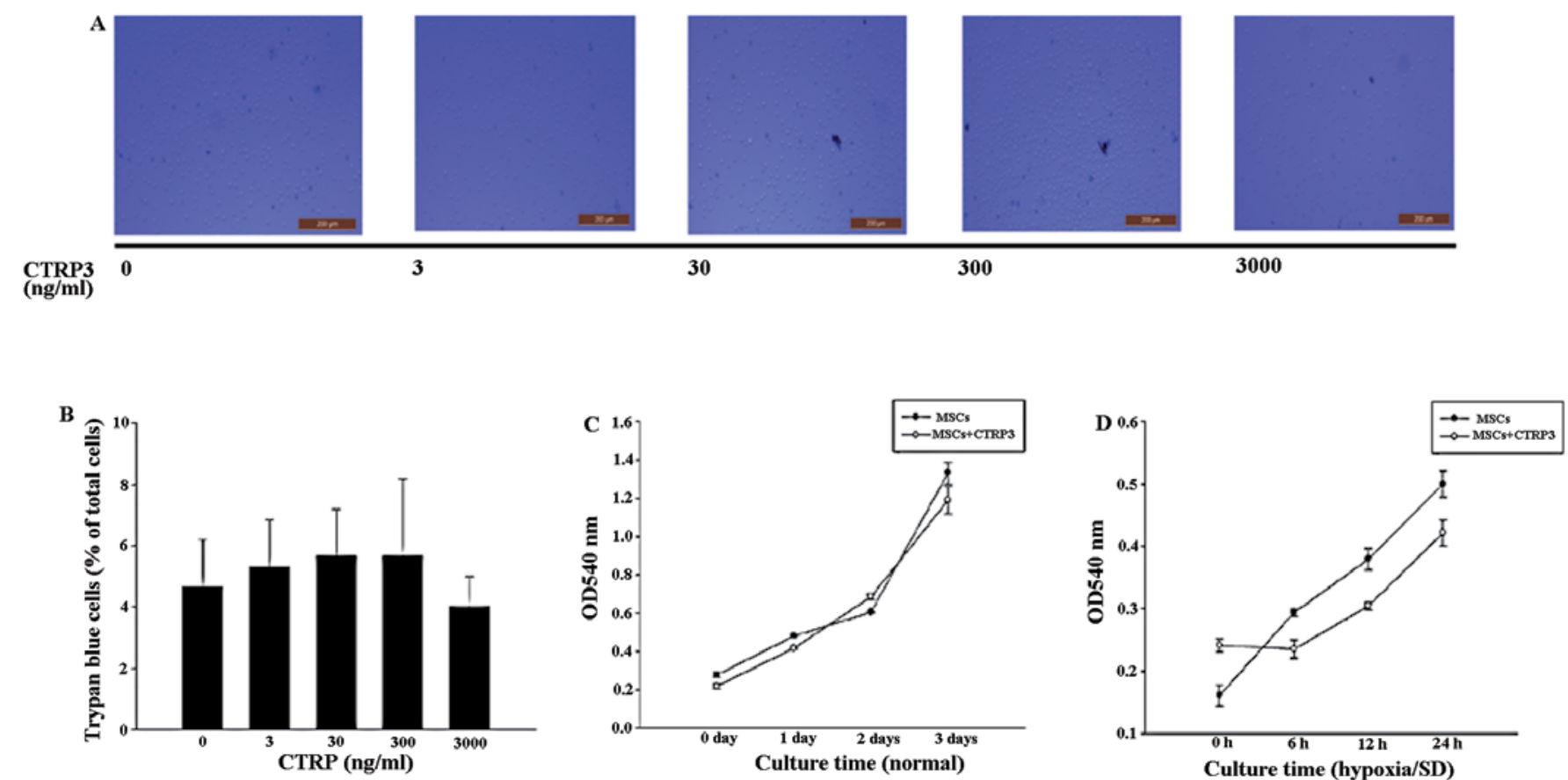

Figure 3. Effects of $\mathrm{Clq}$ tumor necrosis factor-related protein 3 (CTRP3) on cell viability and proliferation. CTRP3 was added to the culture medium to determine its effects on cell viability and the proliferation of mesenchymal stem cells (MSCs). (A and B) Trypan blue staining experiments indicated that up to 3,000 ng/ml of CTRP3 had no toxic effects on cultured MSCs. Each column in (B) represents the mean \pm SD of 3 independent experiments. (C and D) Proliferation growth curves of MSCs were measured by CCK-8 assays. CTRP3 $(300 \mathrm{ng} / \mathrm{ml})$ had no significant effect on the cell proliferation rate during the 3 days of treatment (C) under normal or (D) under 24-h hypoxia/SD culture conditions. Each data point represents the mean \pm SD of 3 independent experiments.

Apoptosis occurred over several hours, and we therefore measured cell proliferation, which could interfere with the measurements of the apoptotic process. The cell proliferation rate, as assessed by CCK- 8 assay, showed no significant change during the 3 days of treatment with $300 \mathrm{ng} / \mathrm{ml}$ CTRP3, either under normal culture or under 24-h hypoxia/SD culture conditions (Fig. 3C and D).

CTRP3 protects MSCs from hypoxia/SD-induced apoptosis through the PI3K/Akt pathway. The PI3K/Akt signaling pathway is considered to be important in promoting survival in numerous cell types (21-23). We therefore investigated the potential involvement of this pathway in the anti-apoptotic effects of CTRP3 in MSCs. Western blot analysis revealed low detectable levels of phospho-Akt in the untreated control cells. Akt phosphorylation was increased by CTRP 3 in a time-dependent manner, peaking at $60 \mathrm{~min}$ [Akt (Ser473) $60 \mathrm{~min}, 4.57 \pm 0.16$ vs. $0 \mathrm{~min}, 1.00 \pm 0.00$; Akt (Tyr308) $60 \mathrm{~min}$, $4.47 \pm 0.33$ vs. $0 \mathrm{~min}, 1.00 \pm 0.00$ ] and remained detectable for up to $90 \mathrm{~min}$ (Fig. 4A and B). To determine the role of the $\mathrm{PI} 3 \mathrm{~K} / \mathrm{Akt}$ signaling pathway in the CTRP3-induced protective effects against hypoxia/SD, MSCs were treated with the specific PI3K/Akt inhibitor, LY294002. The CTRP3-induced phosphorylation of Akt was inhibited by LY294002 $(25 \mu \mathrm{M})$ [Akt (Ser473) with LY294002, 22.65 2.51 vs. without LY294002, 73.20 $\pm 3.02 \%$; Akt (Tyr308) with LY294002, $18.79 \pm 1.09$ vs. without LY294002, 63.47 $\pm 3.21 \%$ ] (Fig. 4C and D). In addition, FACS analysis revealed that LY294002 $(25 \mu \mathrm{M})$ completely blocked the anti-apoptotic effects of CTRP3 (with LY294002, 6.56 \pm 0.11 vs. without LY294002, $3.13 \pm 0.56$ ) (Fig. 4E and F).
CTRP3 exerts anti-apoptotic effects by inhibiting the activation of the mitochondrial pathway. To determine whether CTRP3 exerts its anti-apoptotic effects by inhibiting the activation of the mitochondrial pathway under hypoxia/SD conditions, we examined its effects on the Bcl-2/Bax ratio, the release of cytochrome $c$, caspase- 3 activation and the mitochondrial membrane potential. CTRP3 increased the $\mathrm{Bcl}-2 / \mathrm{Bax}$ ratio (with treatment, $3.07 \pm 0.12$ vs. without treatment, 0.43 \pm 0.09 ) (Fig. 5A), inhibited the release of cytochrome $c$ (without treatment, $0.51 \pm 0.04$ vs. with treatment, $2.95 \pm 0.06$ ) (Fig. 5B) and inhibited the activation of caspase-3 (with treatment, $2.44 \pm 0.18$ vs. without treatment, $5.81 \pm 0.28$ ) (Fig. 5C). In a parallel experiment, CTRP3 reversed the decrease in mitochondrial membrane potential that was induced by hypoxia/SD (Fig. 5D). The PI3K/Akt inhibitor, LY294002 $(25 \mu \mathrm{M})$, blocked the inhibitory effects of CTRP3 on the mitochondrial pathway (Fig. 5).

\section{Discussion}

Autologous MSCs can be easily prepared from adult patients and are immunologically safe. They therefore offer great advantages for regenerating and repopulating the injured myocardium and restoring its function when transplanted into ischemic or infarcted hearts (24). However, despite the implantation of large numbers of cells, the ratio of MSC engraftment remains low due to poor cell survival within the ischemic environment that they are introduced into $(6,21)$. The results of the present study suggest that CTRP3 represents a good candidate for protecting MSCs from apoptosis induced by hypoxia/SD; this protective effect is mediated by the acti- 

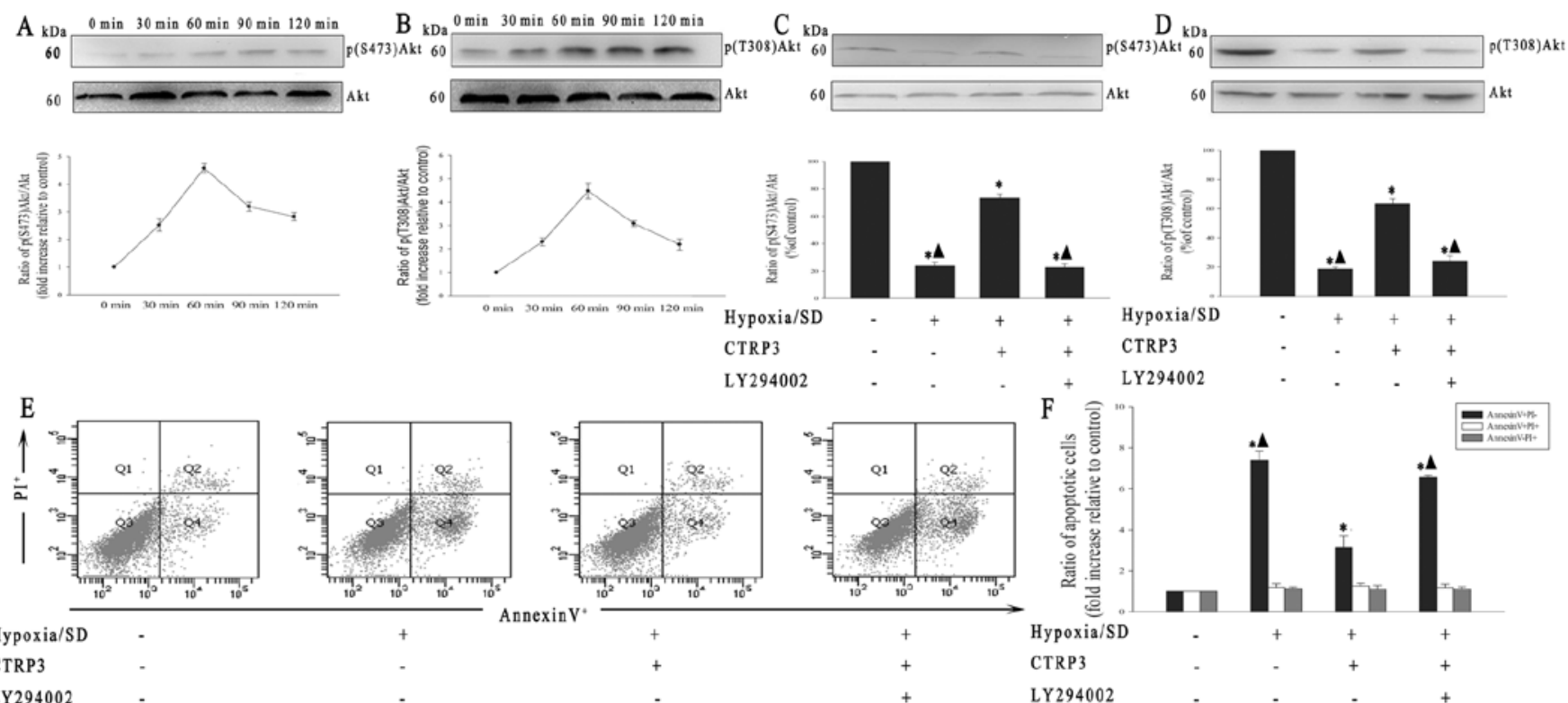

CTRP3

LY294002

-

Figure 4. C1q tumor necrosis factor-related protein 3 (CTRP3) protects mesenchymal stem cells (MSCs) from hypoxia/serum deprivation (SD)-induced apoptosis through the phosphoinositide 3-kinase (PI3K)/Akt pathway. (A and B) MSCs were subjected to hypoxia/SD and stimulated with CTRP3 (300 ng/ml) for the indicated time periods of time. The expression levels of (A) Akt and phospho-Akt (S473), and (B) Akt and phospho-Akt (T308) were analyzed by western blot analysis. Fold changes were estimated relative to Akt. Each data point represents the mean \pm SD of 3 independent experiments. (C and D) Representative western blots of (C) Akt and phospho-Akt [(Ser(S)473], and (D) Akt and phospho-Akt [Tyr(T)308] in cells cultured under hypoxia/SD conditions treated with CTRP3 $(300 \mathrm{ng} / \mathrm{ml}$ ) and pre-treated with LY294002 (a PI3K inhibitor, $25 \mu \mathrm{M}$ ). Fold changes were estimated relative to Akt. Each data column represents the mean \pm SD of 3 independent experiments. To determine the role of the PI3K/Akt pathway in the anti-apoptotic effects of CTRP3, the cells were pre-treated with LY294002 $(25 \mu \mathrm{M})$ for $90 \mathrm{~min}$ in complete medium prior to exposure to hypoxia/SD. CTRP3 $(300 \mathrm{ng} / \mathrm{ml})$ was added in the presence of the inhibitor at the time point of exposure to hypoxia/SD. (E and F) All drugs were maintained in the incubation medium throughout the hypoxia/SD treatment period. Apoptosis was quantified by flow cytometry. Data are presented as fold changes, estimated relative to the corresponding control cells. Each column represents the mean $\pm \mathrm{SD}$ of 3 independent experiments. $(\mathrm{C}, \mathrm{D}$ and $\mathrm{F}){ }^{*} \mathrm{P}<0.05$ vs. control; ${ }^{\wedge} \mathrm{P}<0.05$ vs. hypoxia/SD + CTRP3 (300 ng/ml).

$\mathrm{A}_{\mathrm{k}}$

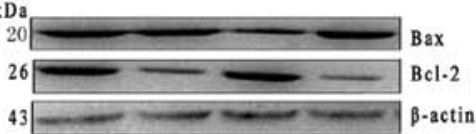

B
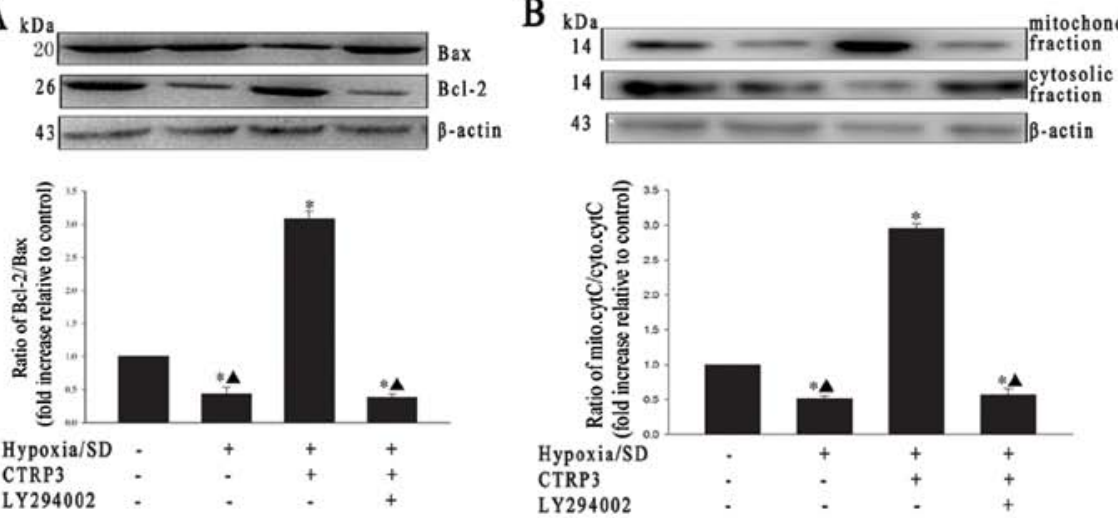

D

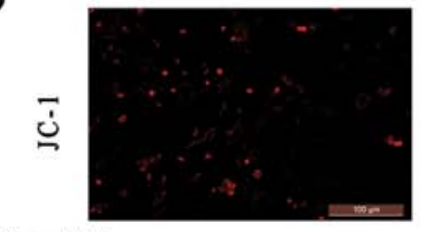

Hypoxia/SD

CTRP3

CTRP3
LY294002
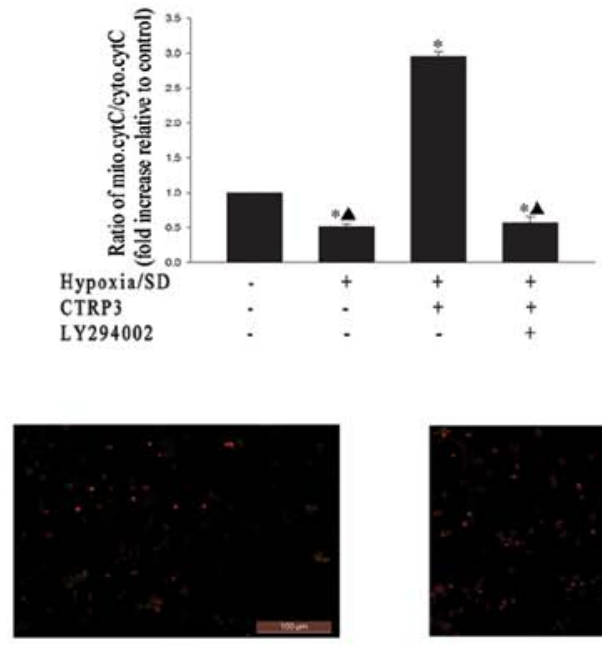

$\mathrm{C}$
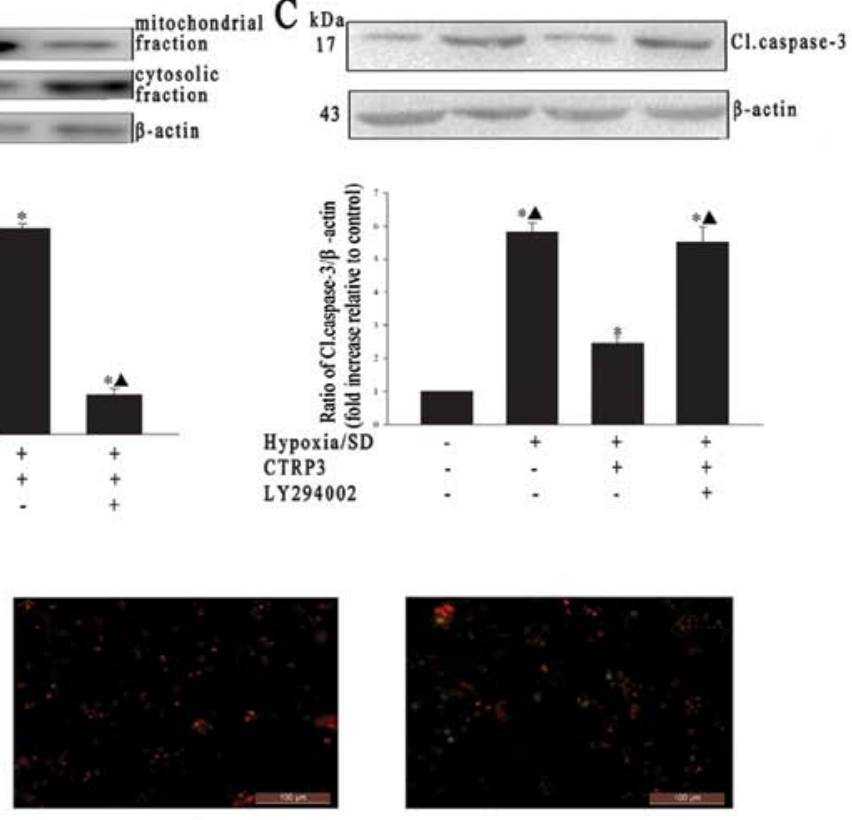

$+$

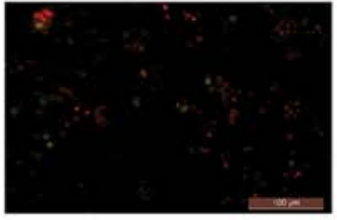

$+$

Figure 5. Clq tumor necrosis factor-related protein 3 (CTRP3) exerts anti-apoptotic effects by inhibiting mitochondrial dysfunction. Mesenchymal stem cells (MSCs) were treated with hypoxia/serum deprivation (SD) for $24 \mathrm{~h}$. In a parallel experiment, cells were pre-treated with LY294002 [a phosphoinositide 3-kinase (PI3K) inhibitor, $25 \mu \mathrm{M}$ ] for $90 \mathrm{~min}$ prior to exposure to hypoxia/SD for $24 \mathrm{~h}$. CTRP3 (300 ng/ml) was added at the time point of exposure to hypoxia/SD. All drugs were maintained in the incubation medium throughout the hypoxia/SD treatment period. (A) Bcl-2/Bax, (B) cytochrome $c$ (mito.cytC, mitochondrial cytochrome $c$; cyto.cytC, cytosolic cytochrome $c$ ), and $(\mathrm{C})$ caspase-3 levels were detected by western blot analysis. Each blot is representative of 3 experiments. Fold changes were estimated relative to $\beta$-actin. Each column represents the mean $\pm \mathrm{SD}$ of 3 independent experiments. ${ }^{*} \mathrm{P}<0.05$ vs. control; ${ }^{\wedge} \mathrm{P}<0.05$ vs. hypoxia/SD + CTRP3 (300 ng/ml). Cl. caspase-3, cleaved caspase-3. (D) Mitochondrial membrane potential visualized by JC-1 staining. 
vation of the PI3K/Akt signaling pathway and the inhibition of the mitochondrial apoptotic pathway.

CTRP3 is a key member of the family of adipokines and has broad functions, not only in adipokine secretion and metabolism, but also in inflammation, cell proliferation, differentiation, apoptosis and cardiac protection (13-17). Recent studies have found that the plasma levels of CTRP3 were decreased in patients with cardiac infarction. In addition, the replenishment of CTRP3 has been shown to attenuate cardiac remodeling and dysfunction and to protect cardiomyocytes from apoptosis in a model of cardiac infarction (13-17). It is possible that the replenishment of CTRP3 in the infarcted myocardium may offer cardioprotection through its ability to influence cell survival, and could potentially enhance MSC survival through the activation of pro-survival signaling pathways. As regards apoptosis, CTRPs have been confirmed to exert strong antiapoptotic effects in various types of cells $(17,25)$, and have been shown to protect cardiomyocytes from apoptosis in cardiac infarction through the PI3K/Akt pathway (17). The PI3K/Akt pathway further displays significant anti-apoptotic effects in MSCs $(20,22,23)$. We thus hypothesized that CTRP3 may protect MSCs from apoptosis induced by hypoxia/SD.

In preliminary experiments, early apoptosis in MSCs induced by hypoxia/SD peaked at $24 \mathrm{~h}$. Using the same model, we found that CTRP3 protected MSCs from apoptosis in a concentration-dependent manner. However, CTRP3 only inhibited the early hypoxia/SD-induced apoptosis of MSCs, and had no effect on late apoptosis or necrosis. CTRP3 had no apparent effect on MSC proliferation, thus eliminating the potential interference between the effects of proliferation and apoptosis.

In the present study, we examined the role of the PI3K/Akt pathway in MSC survival and in modulating the anti-apoptotic effects of CTRP3. PI3K/Akt is an important pro-survival pathway, and the phosphorylation of Akt plays a major role in anti-apoptotic functions in various types of cells (26-29). CTRP3 has been shown to protect cardiomyocytes from apoptosis through the PI3K/Akt pathway (17), and this pathway has been shown to exert a significant protective effect against MSC apoptosis (21-23). Our results revealed that CTRP-3 stimulated Akt phosphorylation and prevented MSCs from hypoxia/SD-induced apoptosis, while inhibition experiments with the PI3K inhibitor, LY294002, indicated that CTRP3 protected MSCs from apoptosis via the PI3K/Akt pathway.

A previous study demonstrated that hypoxia/SD induces apoptosis in MSCs through the mitochondrial apoptotic pathway (19). The present study demonstrated that this process canbeinhibited by CTRP3, which inhibitedhypoxia/SD-induced mitochondrial-dependent apoptosis by increasing the $\mathrm{Bcl}-2 / \mathrm{Bax}$ ratio and the mitochondrial membrane potential, and by inhibiting the release of cytochrome $c$ and the activation of caspase-3; these effects were mediated by the PI3K/Akt pathway. The above results were confirmed by the fact that the effects of CTRP3 on the mitochondrial apoptotic pathway were blocked by the PI3K inhibitor, LY294002. The finding that CTRP3 affected early apoptosis, but had no effect on late apoptosis or necrosis, is in accordance with a previous study that demonstrated the involvement of the mitochondrial apoptotic pathway in the early apoptotic process (30).

In conclusion, the results of the present study suggest that CTRP3 promotes MSC survival under conditions mimicking the ischemic myocardium. CTRP3 protects MSCs from hypoxia/SD-induced mitochondrial apoptosis through the PI3K/Akt signaling pathway. These findings highlight a potential novel therapeutic strategy for protecting MSCs from apoptosis, and form the basis for the future clinical exploitation of CTRP3 and MSCs in cardiac regeneration therapies.

\section{Acknowledgements}

We thank Dr Wei Liu for her expert assistance with the experimental design and excellent technical assistance, and Dr Bo Sun for her assistance with the FACS analysis. Dr Wei Liu and Dr Bo Sun are members of the Key Laboratory of Myocardial Ischemia Mechanism and Treatment (Harbin Medical University), Ministry of Education. This study was supported by grants from the Key Laboratory of Myocardial Ischemia, Harbin Medical University, Ministry of Education (no. KF201315).

\section{References}

1. Lopez AD, Mathers CD, Ezzati M, Jamison DT and Murray CJ: Global and regional burden of disease and risk factors, 2001: systematic analysis of population health data. Lancet 367 : 1747-1757, 2006.

2. Orlic D, Kajstura J, Chimenti S, et al: Bone marrow cells regenerate infarcted myocardium. Nature 410: 701-705, 2001.

3. Stamm C, Westphal B, Kleine HD, et al: Autologous bonemarrow stem-cell transplantation for myocardial regeneration. Lancet 361: 45-46, 2003.

4. Wollert KC, Meyer GP, Lotz J, et al: Intracoronary autologous bone-marrow cell transfer after myocardial infarction: the BOOST randomised controlled clinical trial. Lancet 364 : 141-148, 2004.

5. Zhang M, Methot D, Poppa V, Fujio Y, Walsh K and Murry CE: Cardiomyocyte grafting for cardiac repair: graft cell death and anti-death strategies. J Mol Cell Cardiol 33: 907-921, 2001.

6. Geng YJ: Molecular mechanisms for cardiovascular stem cell apoptosis and growth in the hearts with atherosclerotic coronary disease and ischemic heart failure. Ann NY Acad Sci 1010: 687-697, 2003.

7. Chao W, Shen Y, Li L and Rosenzweig A: Importance of FADD signaling in serum deprivation- and hypoxia-induced cardiomyocyte apoptosis. J Biol Chem 277: 31639-31645, 2002.

8. Bonavita F, Stefanelli C, Giordano E, et al: H9c2 cardiac myoblasts undergo apoptosis in a model of ischemia consisting of serum deprivation and hypoxia: inhibition by PMA. FEBS Lett 536: 85-91, 2003.

9. Kishore U, Gaboriaud C, Waters P, et al: C1q and tumor necrosis factor superfamily: modularity and versatility. Trends Immunol 25: 551-561, 2004.

10. Wong GW, Wang J, Hug C, Tsao TS and Lodish HF: A family of Acrp30/adiponectin structural and functional paralogs. Proc Natl Acad Sci USA 101: 10302-10307, 2004.

11. Schaffler A, Ehling A, Neumann E, et al: Genomic organization, promoter, amino acid sequence, chromosomal localization, and expression of the human gene for CORS-26 (collagenous repeatcontaining sequence of $26-\mathrm{kDa}$ protein). Biochim Biophys Acta 1630: 123-129, 2003.

12. Weigert J, Neumeier M, Schaffler A, et al: The adiponectin paralog CORS-26 has anti-inflammatory properties and is produced by human monocytic cells. FEBS Lett 579: 5565-5570, 2005.

13. Compton SA and Cheatham B: CTRP-3: blocking a toll booth to obesity-related inflammation. Endocrinology 151: 5095-5097, 2010.

14. Kopp A, Bala M, Buechler C, et al: C1q/TNF-related protein-3 represents a novel and endogenous lipopolysaccharide antagonist of the adipose tissue. Endocrinology 151: 5267-5278, 2010.

15. Kopp A, Bala M, Weigert J, et al: Effects of the new adiponectin paralogous protein CTRP-3 and of LPS on cytokine release from monocytes of patients with type 2 diabetes mellitus. Cytokine 49: $51-57,2010$ 
16. Hofmann C, Chen N, Obermeier F, et al: C1q/TNF-related protein-3 (CTRP-3) is secreted by visceral adipose tissue and exerts antiinflammatory and antifibrotic effects in primary human colonic fibroblasts. Inflamm Bowel Dis 17: 2462-2471, 2011.

17. Yi W, Sun Y, Yuan Y, et al: C1q/tumor necrosis factor-related protein-3, a newly identified adipokine, is a novel antiapoptotic, proangiogenic, and cardioprotective molecule in the ischemic mouse heart. Circulation 125: 3159-3169, 2012.

18. Pittenger MF, Mackay AM, Beck SC, et al: Multilineage potential of adult human mesenchymal stem cells. Science 284 143-147, 1999.

19. Zhu W, Chen J, Cong X, Hu S and Chen X: Hypoxia and serum deprivation-induced apoptosis in mesenchymal stem cells. Stem Cells 24: 416-425, 2006.

20. Chen J, Baydoun AR, Xu R, et al: Lysophosphatidic acid protects mesenchymal stem cells against hypoxia and serum deprivationinduced apoptosis. Stem Cells 26: 135-145, 2008.

21. Mangi AA, Noiseux N, Kong D, et al: Mesenchymal stem cells modified with Akt prevent remodeling and restore performance of infarcted hearts. Nat Med 9: 1195-1201, 2003.

22. Hahn JY, Cho HJ, Kang HJ, et al: Pre-treatment of mesenchymal stem cells with a combination of growth factors enhances gap junction formation, cytoprotective effect on cardiomyocytes and therapeutic efficacy for myocardial infarction. J Am Coll Cardiol 51: 933-943, 2008.

23. Lu G, Haider HK, Jiang S and Ashraf M: Sca- $1^{+}$stem cell survival and engraftment in the infarcted heart: dual role for preconditioning-induced connexin-43. Circulation 119: 2587-2596, 2009.
24. Orlic D, Kajstura J, Chimenti S, et al: Mobilized bone marrow cells repair the infarcted heart, improving function and survival. Proc Natl Acad Sci USA 98: 10344-10349, 2001.

25. Li Q, Wang L, Tan W, et al: Identification of ClqTNF-related protein 4 as a potential cytokine that stimulates the STAT3 and $\mathrm{NF}-\kappa \mathrm{B}$ pathways and promotes cell survival in human cancer cells. Cancer Lett 308: 203-214, 2011.

26. Zhu P, Tan MJ, Huang RL, et al: Angiopoietin-like 4 protein elevates the prosurvival intracellular $\mathrm{O}_{2}(-): \mathrm{H}_{2} \mathrm{O}_{2}$ ratio and confers anoikis resistance to tumors. Cancer Cell 19: 401-415, 2011.

27. Albrecht-Schgoer K, Schgoer W, Holfeld J, et al: The angiogenic factor secretoneurin induces coronary angiogenesis in a model of myocardial infarction by stimulation of vascular endothelial growth factor signaling in endothelial cells. Circulation 126: 2491-2501, 2012

28. Kanwar JR, Kamalapuram SK and Kanwar RK: Survivin signaling in clinical oncology: a multifaceted dragon. Med Res Rev 33: 765-789, 2013.

29. Shortt J, Martin BP, Newbold A, et al: Combined inhibition of PI3K-related DNA damage response kinases and mTORC1 induces apoptosis in MYC-driven B-cell lymphomas. Blood 121: 2964-2974, 2013.

30. Xu R, Chen J, Cong X, Hu S and Chen X: Lovastatin protects mesenchymal stem cells against hypoxia- and serum deprivationinduced apoptosis by activation of PI3K/Akt and ERK1/2. J Cell Biochem 103: 256-269, 2008. 